\title{
Expression of the cannabinoid type I receptor and prognosis following surgery in colorectal cancer
}

\author{
CHAN KWON JUNG ${ }^{1 *}$, WON KYUNG KANG ${ }^{2 *}$, JAE MYUNG PARK ${ }^{3}$, HYO JUN AHN $^{3}$, \\ SANG WOO $\mathrm{KIM}^{3}$, SEONG TAEK $\mathrm{OH}^{1}$ and $\mathrm{KYU}$ YONG $\mathrm{CHOI}^{3}$ \\ Departments of ${ }^{1}$ Hospital Pathology, ${ }^{2}$ Surgery and ${ }^{3}$ Internal Medicine, College of Medicine, \\ The Catholic University of Korea, Seoul 137-070, Republic of Korea
}

Received September 26, 2012; Accepted December 14, 2012

DOI: $10.3892 / \mathrm{ol} .2012 .1081$

\begin{abstract}
The cannabinoid system has been considered to be a potential target of colorectal carcinoma therapy. The aim of this study was to address the correlation between cannabinoid type 1 (CB1) receptor expression and disease severity/outcomes in patients with colorectal cancer (CRC). CB1 receptor expression was analyzed by immunohistochemistry using tissue microarrays in consecutive patients who underwent surgical resection $(\mathrm{n}=534)$. CB1 receptor expression was categorized as a high $(\geq 66 \%)$ vs. low $(<66 \%)$ immunopercentage as a median split, and was analyzed in relation to disease severity and overall survival. CB1 receptor expression was observed in 409 patients (76.6\%). Low CB1 receptor expression was more frequently identified in stage IV than in stage I/II or III cancer $(\mathrm{P}<0.01$ for both). In stage IV CRC, high vs. low CB1 expression was correlated with a statistically significant poorer overall survival $(\mathrm{P}=0.033)$ that was independent of age, $\mathrm{R} 0$ resection, tumor differentiation and chemotherapy [hazard ratio (HR), 1.805; 95\% confidence interval (CI), 1.042-3.094; $\mathrm{P}=0.035]$. However, $\mathrm{CB} 1$ expression was not observed to be correlated with patient survival following surgery in stage I/II or III cancer. The high immunoreactivity of the cannabinoid type 1 receptor is a significant prognostic factor following surgery in stage IV CRC.
\end{abstract}

\section{Introduction}

Cannabinoids have been implicated in physiological and pathological conditions including inflammation, immunity,

Correspondence to: Dr Jae Myung Park, Department of Internal Medicine, College of Medicine, The Catholic University of Korea, No. 505 Banpo-Dong, Seocho-Gu, Seoul 137-701, Republic of Korea E-mail: parkjerry@catholic.ac.kr

*Contributed equally

Key words: cannabinoid receptor, survival, Kaplan-Meier analysis, stage, metastasis analgesia, neoplasia and others (1). These associations have increased the interest in cannabinoids in recent years. The effect of cannabinoids in colorectal cancer (CRC) has been demonstrated in in vitro experiments and animal models, which indicate the antiproliferative, apoptotic and antimetastatic actions of cannabinoid agonists (2-5). In accordance with these observations, the levels of the major endogenous cannabinoids have been identified to be 2- to 3-fold higher in $\mathrm{CRC}$ than in the neighboring normal mucosa (5).

Antineoplastic effects are mediated by the activation of cannabinoid type I (CB1), type 2 (CB2) or a non-cannabinoid receptor-mediated mechanism (6). Among the receptors, the mechanism of tumor cell apoptosis has been rigorously investigated by several research groups who have studied the CB1 receptor (2-5). CB1 is abundantly expressed in the brain and in numerous peripheral neurons (7). It is also found not only in normal colonic epithelium, smooth muscle and the submucosal myenteric plexus (8), but also in several colon cancer cell lines (6). Expression levels of CB1 receptors are downregulated in cancer compared with adjacent normal mucosa (3). Loss or inhibition of the CB1 receptor has been demonstrated to accelerate intestinal adenoma growth, whereas activation of the $\mathrm{CB} 1$ receptor attenuated intestinal tumor growth by inducing cell death via downregulation of the anti-apoptotic factor, survivin, in a genetic model of CRC progression (3). CRC patients who are either homo- or heterozygous for the $1359 \mathrm{G} / \mathrm{A} \mathrm{CB} 1$ receptor polymorphism exhibit a shorter survival time compared with $\mathrm{G} / \mathrm{G}$ wild-type patients, although the post-transcriptional mechanism has not yet been delineated (9).

The evidence mentioned thus far suggests that the endogenous cannabinoid system is able to regulate cancer progression and affect disease progression and outcomes. Although there have been a small number of studies concerning the prognostic role of the CB1 receptor in human tissues (10-12), the results were discrepant and CB1 expression of CRC has only been addressed in a single study (13). We hypothesize that increased CB1 receptor expression may be associated with decreased disease severity and more favorable clinical outcomes. Therefore, the aim of the present study was to investigate the correlation between disease severity/clinical outcomes and the expression level of the CB1 receptor. 


\section{Materials and methods}

Patients. Between January, 2004 and December, 2007, a total of 544 consecutive patients with CRC who underwent surgery at Seoul St. Mary's Hospital, Seoul, Korea, were enrolled. Patients who had surgery-related mortality $(n=10)$ were excluded from this study; therefore, the clinical data of 534 patients were analyzed. The patients were followed up after surgery at regular 3- to 6-month intervals during the first year and then at 6-month intervals thereafter. Abdominal computed tomography was performed annually for the first 3 years and colonoscopy was performed every 1-3 years for evaluation of recurrence. The follow-up time for patients who did not survive was defined as the duration between the dates of surgery and mortality. The Institutional Review Board at Seoul St. Mary's Hospital approved the handling of tissue samples and the patient data in the present study. Written informed consent was obtained from the patients and patient anonymity was preserved throughout this study.

The mean patient age was 62.8 years (standard deviation, $11.7)$ and 328 of the patients $(61.4 \%)$ were male. The tumor location was the right colon in $150(28.1 \%)$ patients, the left colon in 178 patients (33.3\%) and the rectum in 206 patients (38.6\%). The numbers of patients with disease stages I, II, III and IV were 78 (14.6\%), 162 (30.3\%), 206 (38.6\%) and 88 (16.5\%), respectively, according to the American Joint Committee on Cancer tumor node metastasis (TNM) system (14). The tumor grade was well/moderately differentiated in $494(92.5 \%)$ patients and poorly differentiated in $40(7.5 \%)$ patients, by the World Health Organization tumor classifcation system (15). R0 resection was administered to $478(89.5 \%)$ patients, and $97(18.2 \%)$ patients received adjuvant chemotherapy. The median follow-up time was 42 months (range, 2-80). All data regarding the follow-up studies were evaluated on the basis of information available as of October, 2010.

Tissue microarray generation. Tissue microarrays were constructed from archival formalin-fixed, paraffin-embedded carcinoma samples obtained from primary CRC specimens using a manual tissue arrayer (Quick-Ray Manual Tissue Microarrayer; Unitma Co., Ltd.; Seoul, Korea). For each sample, two areas rich in viable tumor cells $(>80 \%)$ in the invasive front and in the tumor center were identified by light microscopic examination of hematoxylin and eosin-stained sections, and were marked for use in the tissue microarrays. Tissue cylinders with a diameter of $2 \mathrm{~mm}$ were punched from the previously marked tumor area of each block (the donor block) and inserted into a recipient paraffin block, resulting in a $6 \times 10$ array.

Immunohistochemical staining. Immunohistochemistry for CB1 was performed on paraffin-embedded tissue sections of the tissue microarrays. Immunohistochemical reactions were conducted using a Polink-2 HRP Plus Broad detection system (Golden Bridge International; Mukilteo, WA, USA) according to the manufacturer's instructions. Briefly, the tissue sections were deparaffinized and quenched with $3 \%$ hydrogen peroxide in methanol for $10 \mathrm{~min}$. Antigen retrieval was then conducted using $0.01 \mathrm{~mol} / 1$ citrate buffer ( $\mathrm{pH}$ 6.0) by heating the sample in a microwave pressure cooker for
20 min. The sections were incubated with rabbit polyclonal CB1 antibody (1:50; Cat. No. 23703; Abcam; Cambridge, UK) $(10,13)$ at room temperature for $30 \mathrm{~min}$, followed by incubation with Broad Antibody Enhancer for $10 \mathrm{~min}$ and then with Polymer-HRP for 10 min (Golden Bridge International, Inc., Mukilteo, WA, USA). The peroxidase reaction was developed using 3'3-diaminobenzidine tetrahydrochloride as the chromogen. Negative controls included substitution of the primary antibodies with normal rabbit IgG of the same concentration as the primary antibodies.

Scoring of immunohistochemistry. Each preserved specimen was examined by a pathologist (C.K.J.) who was blind to the clinical status of the patients. The immunoreactive area for CB1 was scored as $0(0 \%), 1(<33 \%), 2(33-66 \%)$ or $3(>66 \%)$, as discussed previously by Michalski et al (11). Using a simple median split, i.e., $<3$ or $=3$, immunoreactivity of CB1 expression was categorized as low or high under light microscopy. When the pathologist had scored all the samples, these were repeatedly measured using the same procedure, but without accessing any previous data. Cases with different scores were then evaluated once more, also without knowledge of the previous results. The final scores were then entered into the database for analysis by another investigator (J.M.P.).

Statistical analysis. Continuous data are presented as the mean \pm standard deviation (SD), and categorical data are presented as quantities and proportions. To evaluate the difference between the groups of patients according to the immunoreactivity of CB1 receptor expression, the $\chi^{2}$ test was used for categorical data, and the two-sample independent t-test was used for continuous variables. Cumulative survival curves for patients according to $\mathrm{CB} 1$ immunoreactivity were determined by the Kaplan-Meier method, and differences between the groups were compared using the log-rank test. Univariate and multivariate analyses were performed with the Cox proportional hazard regression model to determine factors related to overall survival. All statistical analyses were performed using Statistical Analysis Software (SAS; SAS Institute; Cary, NC, USA). $\mathrm{P}<0.05$ was considered to indicate a statistically significant difference.

\section{Results}

Immunohistochemical evaluation of CBI expression. Tumor cells with positive cytoplasmic staining for $\mathrm{CB} 1$ are illustrated in Fig. 1. Positive immunoreactivity for the $\mathrm{CB} 1$ receptor was observed in 409 patients (76.6\%). The area of immunoreactive tumor cells was zero in 125 patients $(23.4 \%),<33 \%$ in 24 patients (4.5\%), 33-66\% in 114 patients (21.3\%) and $>66 \%$ in 271 patients $(50.7 \%)$. The proportion of low expression was significantly higher in stage IV than in stage I/II or III cancer $(\mathrm{P}<0.01$ for both; Fig. 2). The expression level of the $\mathrm{CB} 1$ receptor at the invasive front was similar to that in the specimens from the tumor center $(\mathrm{P}=\mathrm{NS})$.

Association of CB1 expression with clinicopathological findings. The correlations between CB1 receptor expression and the clinicopathological characteristics are summarized in Table I. No significant differences were observed in age, 
Table I. Characteristics of the tumor tissue samples with high and low expression of the cannabinoid type I (CB1) receptor.

\begin{tabular}{|c|c|c|c|}
\hline \multirow[b]{2}{*}{ Categories } & \multicolumn{2}{|c|}{ Expression of the $\mathrm{CB} 1$ receptor } & \multirow[b]{2}{*}{ P-value } \\
\hline & Low $(\mathrm{n}=263)(\%)$ & $\operatorname{High}(\mathrm{n}=271)(\%)$ & \\
\hline Mean age \pm SD (years) & $62.3 \pm 11.7$ & $63.3 \pm 11.7$ & 0.319 \\
\hline \multicolumn{4}{|l|}{ Gender } \\
\hline Male & $163(62.0)$ & $165(60.9)$ & \multirow[t]{2}{*}{0.796} \\
\hline Female & $100(38.0)$ & $106(39.1)$ & \\
\hline Tumor size & $5.0 \pm 1.8$ & $5.4 \pm 2.4$ & 0.168 \\
\hline \multicolumn{4}{|c|}{ Histological differentiation ${ }^{\mathrm{a}}$} \\
\hline Differentiated & $245(93.2)$ & 249 (91.9) & \multirow[t]{2}{*}{0.576} \\
\hline Undifferentiated & $18(6.8)$ & $22(8.1)$ & \\
\hline \multicolumn{4}{|l|}{ Tumor site } \\
\hline Right & $71(27.0)$ & $79(29.2)$ & \multirow[t]{3}{*}{0.157} \\
\hline Left & $80(30.4)$ & $98(36.2)$ & \\
\hline Rectum & $112(42.6)$ & $94(34.7)$ & \\
\hline \multicolumn{3}{|l|}{ Depth of invasion } & \multirow[t]{5}{*}{0.888} \\
\hline $\mathrm{T} 1$ & $7(2.7)$ & $8(3.0)$ & \\
\hline $\mathrm{T} 2$ & $32(12.2)$ & $39(14.4)$ & \\
\hline $\mathrm{T} 3$ & $167(63.5)$ & $167(61.6)$ & \\
\hline $\mathrm{T} 4$ & $57(21.7)$ & $57(21.0)$ & \\
\hline \multicolumn{4}{|l|}{ Lymph node metastasis } \\
\hline No & $119(45.2)$ & $137(50.6)$ & \multirow[t]{3}{*}{0.112} \\
\hline N1 & $67(25.5)$ & $76(28.0)$ & \\
\hline $\mathrm{N} 2$ & $77(29.3)$ & $58(21.4)$ & \\
\hline \multicolumn{4}{|l|}{ Distant metastasis } \\
\hline M0 & $207(78.7)$ & $239(88.2)$ & \multirow[t]{2}{*}{0.003} \\
\hline M1 & $56(21.3)$ & $32(11.8)$ & \\
\hline \multicolumn{3}{|l|}{ TNM stage } & \multirow[t]{5}{*}{0.023} \\
\hline I & $36(13.7)$ & $42(15.5)$ & \\
\hline II & $71(27.0)$ & $91(33.6)$ & \\
\hline III & $100(38.0)$ & $106(39.1)$ & \\
\hline IV & $56(21.3)$ & $32(11.8)$ & \\
\hline
\end{tabular}

an cases of mixed histological differentiation, the more poorly differentiated tumor was selected. TNM, tumor node metastasis.
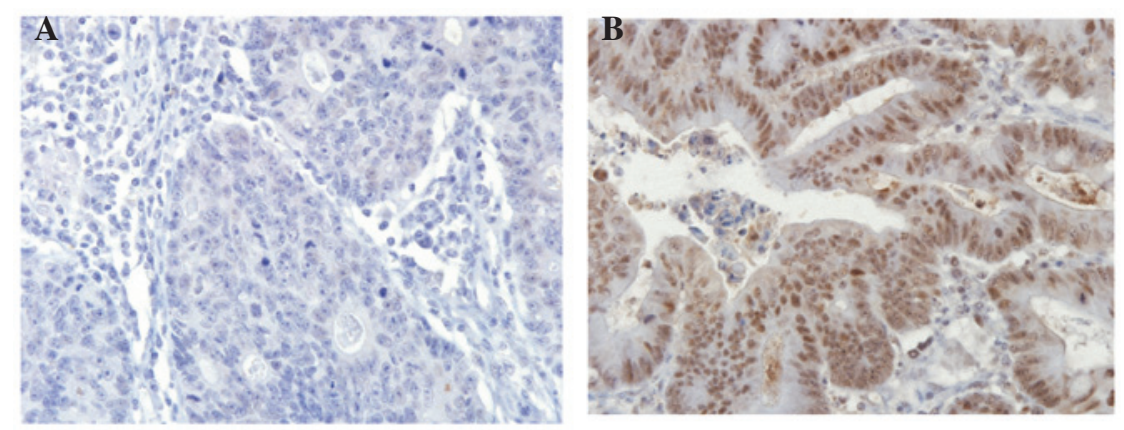

Figure 1. Examples of immunohistochemical staining of the cannabinoid type 1 (CB1) receptor in human colorectal cancer. Tumor cells exhibit low (A) and high (B) expression of the CB1 receptor. Original magnification, $\mathrm{x} 400$.

gender, tumor size, histological differentiation, primary tumor site, depth of invasion and lymph node metastasis between high and low CB1 receptor expression in the immunostaining results. However, distant metastasis was significantly higher in 
Table II. Clinicopathological characteristics of patients with stage IV colorectal cancer in relation to expression of cannabinoid type I (CB1) receptor immunoreactivity.

Expression of the $\mathrm{CB} 1$ receptor

\begin{tabular}{|c|c|c|c|}
\hline \multirow[b]{2}{*}{ Categories } & & \multirow[b]{2}{*}{ P-value } \\
\hline & Low $(\mathrm{n}=56)(\%)$ & $\operatorname{High}(\mathrm{n}=32)(\%)$ & \\
\hline Mean age \pm SD (years) & $59.1 \pm 11.9$ & $61.5 \pm 9.3$ & 0.293 \\
\hline \multicolumn{4}{|l|}{ Gender } \\
\hline Male & $34(60.7)$ & $20(62.5)$ & 0.869 \\
\hline Female & $22(39.3)$ & $12(37.5)$ & \\
\hline Histological differentiation ${ }^{\mathrm{a}}$ & & & 0.210 \\
\hline Differentiated & $48(85.7)$ & $24(75.0)$ & \\
\hline Undifferentiated & $8(14.3)$ & $8(25.0)$ & \\
\hline Tumor site & & & 0.187 \\
\hline Right & $20(35.7)$ & $8(25.0)$ & \\
\hline Left & $17(30.4)$ & $16(50.0)$ & \\
\hline Rectum & $19(33.9)$ & $8(25.0)$ & \\
\hline Adjuvant chemotherapy & $18(32.1)$ & 7 (21.9) & 0.304 \\
\hline R0 resection & $29(51.8)$ & $18(56.3)$ & 0.686 \\
\hline Follow-up (months) & $22.5(15-40.3)$ & $17(8.3-32.3)$ & 0.134 \\
\hline
\end{tabular}

${ }^{a}$ In cases of mixed differentiation, the more poorly differentiated tumor was selected. ${ }^{b}$ Median and interquartile range, compared by Kruskal-Wallis test.

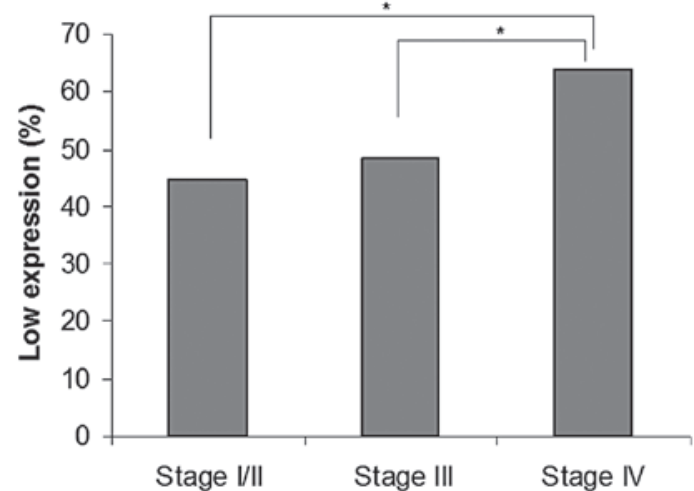

Figure 2. Cannabinoid type 1 (CB1) expression according to tumor stage. Compared to stage I/II or III, low CB1 expression is demonstrated more frequently in stage IV colorectal cancer. ${ }^{*} \mathrm{P}<0.01$.

the patients with low CB1 receptor expression compared with those classified as having high expression $(\mathrm{P}=0.003)$. Accordingly, the frequency was shown to be different in stage IV cancer $(\mathrm{P}=0.023)$.

As the frequency of distant metastasis was different between the group with high and low CB1 receptor expression, we compared the clinicopathological characteristics in stage IV cancer (Table II). No significant differences were observed in age, gender, histological differentiation, tumor site and follow-up interval between high and low CB1 receptor expression. Additionally, no significant difference was observed in the number of R0 resections performed in patients with high and low CB1 receptor expression; 18 (56.3\%) and $29(51.8 \%)$, respectively $(\mathrm{P}=0.686)$.
Survival according to cancer stage. The Kaplan-Meier analysis of the data for a total of 534 patients revealed that the overall survival between the patients with high vs. low CB1 receptor expression in tumors was not significantly different (Fig. 3A) (log-rank test; $\mathrm{P}=0.316$ ). In stage I/II and III cancers, the overall survival of patients with high vs. low $\mathrm{CB} 1$ receptor expression was not significantly different (Fig. 3B and C). The patients with high CB1 receptor expression in tumors had poorer outcomes than the patients with low expression (Fig. 3D). The hazard ratio (HR) was 1.782 with a 95\% confidence interval $(\mathrm{CI})$ of 1.049-2.988 $(\mathrm{P}=0.033)$.

Factors affecting overall survival. The multivariate analysis of factors related to overall survival in stage IV is shown in Table III. A Cox proportional hazards regression analysis indicated that a high CB1 expression level, along with incomplete resection of the tumor and undifferentiated pathology was an independent variable associated with a poorer disease outcome in stage IV cancer (HR, 1.805; 95\% CI, 1.042-3.094; $\mathrm{P}=0.035)$.

\section{Discussion}

The function of the CB1 receptor underlying the pathophysiology and outcome of CRCs has not been clearly delineated. In this study, we demonstrated that $\mathrm{CB} 1$ receptor expression is correlated with distant metastasis, but not with tumor invasion and lymph node metastasis, in CRC. In terms of the patient outcome, high CB1 receptor expression is correlated with poor survival in stage IV CRC, and is an independent prognostic factor even after adjusting for covariates. However, high 
Table III. Multivariate analysis of tumor variables and overall survival in stage IV cancer ( $\mathrm{n}=88)$.

\begin{tabular}{lll}
\hline Variable & \multicolumn{1}{c}{ HR $(95 \%$ CI $)$} & P-value \\
\hline Age & $1.000(0.974-1.021)$ & 0.7803 \\
Expression of CB1 receptor $(\geq 66$ vs. $<66 \%)$ & $1.805(1.042-3.094)$ & 0.0353 \\
Resection (R1/R2 vs. R0) & $4.506(1.933-13.152)$ & 0.0002 \\
Chemotherapy (no vs. yes) & $2.000(0.761-6.213)$ & 0.1653 \\
Differentiation (undifferentiated vs. differentiated) & $2.755(1.449-4.987)$ & 0.0027
\end{tabular}

$\mathrm{CB} 1$, cannabinoid type 1 ; HR, hazard ratio ; CI, confidence interval.

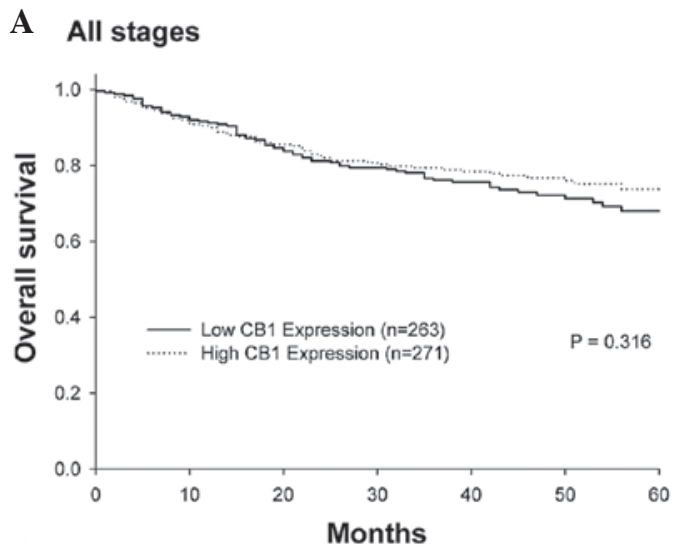

C Stage III

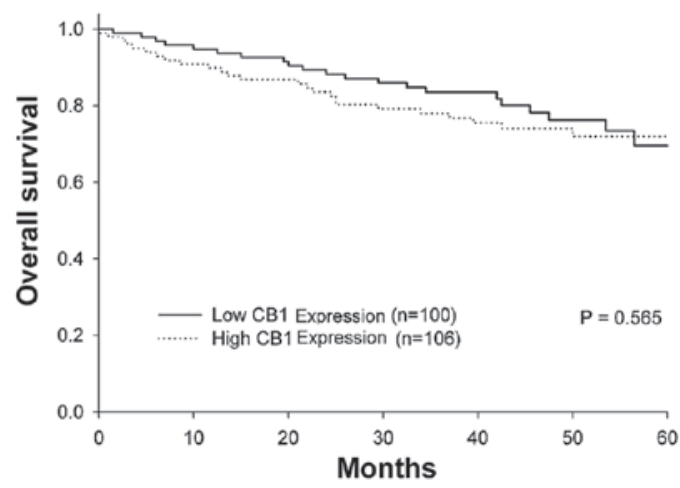

B Stage I/II

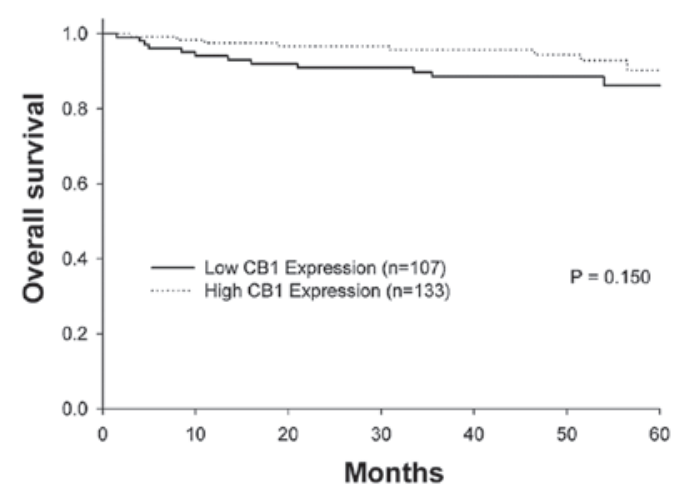

D Stage IV

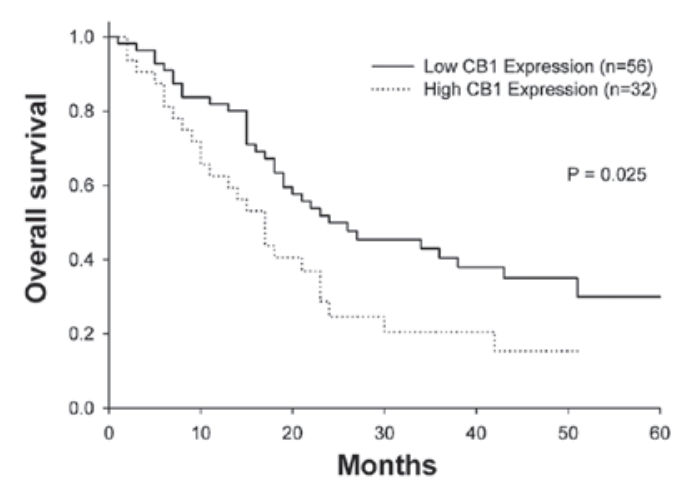

Figure 3. Influence of cannabinoid type 1 (CB1) receptor expression at surgery on overall survival by Kaplan-Meier plots. (A) All stages; (B) stage I/II; (C) stage III and (D) stage IV.

CB1 receptor expression was not associated with the clinical outcomes in stage I/II and III CRC.

Cannabinoid molecules have been of significance in the study of potential cancer therapies, as they have demonstrated potential antitumor effects in cultured cell lines and in animal models. Previous studies have indicated that the majority of the effects of exogenous cannabinoids act through the activation of the CB1 receptor (6). In an endogenous system, downregulation of $\mathrm{CB} 1$ receptor expression was observed in neoplastic epithelial cells from colon cancer biopsies (16). The mechanism of this finding is explained by epigenetic silencing of the CB1-encoding gene that contributes to a loss of its transcription (3), as is frequently found in the inactivation of tumor suppressor genes (17). However, the functions of the $\mathrm{CB} 1$ receptor gene and the mechanisms underlying the transcriptional regulation of the $\mathrm{CB} 1$ receptor are not clearly delineated. The present data revealed that $\mathrm{CB} 1$ receptor expression is downregulated as CRC progresses to a highly advanced stage, which is concordant with our hypothesis. This finding is also supported by a previous observation by Gustafsson et al that $64 \%$ of patients with stage IV CRC exhibited low immunoreactivity of the CB1 receptor (13).

Our data demonstrated that high $\mathrm{CB} 1$ receptor expression in CRC confers a poor prognosis for the patient. We had predicted that high CB1 receptor expression would lead to a better outcome in patients with CRC; however, the opposite result was observed. Notably, our observation was similar to that of the study by Gustafsson et al, in which CRC with a high intensity 
of the CB1 receptor was correlated with a shorter survival time than those with a low CB1 receptor intensity (13). It is difficult to clearly explain this observation. In general, the prognosis of CRC has been known to be correlated with the type, density and location of immune cells within the tumor (18), and certain studies have indicated that the presence of tumor-infiltrating lymphocytes within cancer cell nests and the tumor stroma is related to improved survival $(19,20)$. CB1 has a pivotal role in modulating the immune response. A previous study demonstrated that mice in which the CB1-encoding gene had been knocked out exhibited a stronger inflammatory response in the colon compared with wild-type mice in response to treatment with pro-inflammatory agents (21), suggesting an immunomodulatory role of the $\mathrm{CB} 1$ receptor. Therefore, it may be speculated that tumors with low CB1 expression in the tumor tissue may elicit a low inflammatory environment. The study by Gustafsson et al revealed that $\mathrm{CRC}$ with low $\mathrm{CB} 1$ receptor intensity in the tumor front presented a higher number of infiltrating lymphocytes than CRC with high CB1 receptor intensity, with marginal statistical significance (13). The other possible explanation for this observation is that a high level of CB1 receptor expression is able to activate the pro-survival cellular pathway. CB1 receptors are coupled to a variety of signaling cascades, including cyclic AMP and activation of the extracellular signal-related kinase pathway (7), which are able to cause cell proliferation (22). Furthermore, a previous study indicated that activation of the $\mathrm{CB} 1$ receptor results in activation of the Akt signaling pathway and that cannabinoids only induced tumor cell apoptosis when this pathway was inhibited (23).

Another potential explanation is that $\mathrm{CB} 1$ receptor expression is a compensatory response for the endogenous cannabinoid level. This implies that a higher degree of endogenous cannabinoid in tumors favors apoptosis in cancer cells; this results in better survival and leads to downregulation of CB1 receptor expression in a compensatory way. Regarding this explanation, the level of endogenous cannabinoid-metabolizing enzymes, as well as the CB1 receptor, were analyzed in a pancreatic cancer study; however, this study failed to indicate the correlation between enzyme function and CB1 receptor level and patient survival (11). Studies in CRC to further explain this observation are necessary.

Notably, high CB1 receptor expression in stage IV CRC is an independent prognostic factor, even following adjustment for R0 resection, tumor differentiation and chemotherapy. A recent study indicated that the $\mathrm{CB} 1$ receptor antagonist rimonabant was able to control tumor growth (24). Therefore, it would be valuable to know whether patients with high $\mathrm{CB} 1$ receptor expression can be treated effectively with this receptor-blocking agent.

To our knowledge, four studies have investigated the correlation between CB1 receptor expression and disease outcome in cancer. Our data was supported by three of these studies, which demonstrated that high CB1 receptor immunoreactivity was correlated with a shorter survival time than low immunoreactivity in pancreatic, prostate and colorectal cancers $(10,11,13)$. In contrast, disease-free survival in hepatocellular carcinoma was observed to be lower in patients with low CB1 receptor immunoreactivity than in those with high immunoreactivity (12). These discrepant results may indicate different roles of $\mathrm{CB} 1$ receptors that are dependent on the type of cancer.
The observation time and number of mortalities in our study may not have been sufficient to discern the survival difference according to the CB1 receptor expression level in stage I/II or III CRC; therefore, these factors may have caused different results from a previous study (13). However, we propose that the results of stage IV cancer portray the true role of CB1, as fatalities occurred during a relatively short time period. Furthermore, the multivariate analysis supports the important and independent association of the CB1 receptor with patient survival. In a previous study of patients with pancreatic cancer, which has a poorer prognosis than CRC, a difference in survival was observed according to the expression level of CB1 (11). Limitations of the present study include the status of microsatellite instability, the level of endogenous cannabinoids and the fact that the metabolizing enzymes of endogenous cannabinoids in tumors were not evaluated. The use of a large sample size of well-characterized patients with a long follow-up period may allow for the correlation between CB1 expression and the disease outcomes to be determined.

In conclusion, the present study demonstrated that high $\mathrm{CB} 1$ receptor expression is independently correlated with decreased survival in stage IV CRC. Future studies investigating other components of the endogenous cannabinoid system are required to clarify the exact mechanism and the correlation with endogenous cannabinoids.

\section{Acknowledgements}

This study was supported by the 2010 Research Foundation of the Department of Internal Medicine, The Catholic University of Korea and by the Korea Research Foundation (Grant No. 2009-0066500).

\section{References}

1. Di Marzo V: Targeting the endocannabinoid system: to enhance or reduce? Nat Rev Drug Discov 7: 438-455, 2008.

2. Greenhough A, Patsos HA, Williams AC and Paraskeva C: The cannabinoid delta(9)-tetrahydrocannabinol inhibits RAS-MAPK and PI3K-AKT survival signalling and induces BAD-mediated apoptosis in colorectal cancer cells. Int J Cancer 121: 2172-2180, 2007.

3. Wang D, Wang H, Ning W, Backlund MG, Dey SK and DuBois RN: Loss of cannabinoid receptor 1 accelerates intestinal tumor growth. Cancer Res 68: 6468-6476, 2008.

4. Patsos HA, Hicks DJ, Dobson RR, et al: The endogenous cannabinoid, anandamide, induces cell death in colorectal carcinoma cells: a possible role for cyclooxygenase 2 . Gut 54: 1741-1750, 2005.

5. Ligresti A, Bisogno T, Matias I, et al: Possible endocannabinoid control of colorectal cancer growth. Gastroenterology 125: 677-687, 2003.

6. Izzo AA and Camilleri M: Cannabinoids in intestinal inflammation and cancer. Pharmacol Res 60: 117-125, 2009.

7. Howlett AC, Barth F, Bonner TI, et al: International Union of Pharmacology. XXVII. Classification of cannabinoid receptors. Pharmacol Rev 54: 161-202, 2002.

8. Wright K, Rooney N, Feeney M, et al: Differential expression of cannabinoid receptors in the human colon: cannabinoids promote epithelial wound healing. Gastroenterology 129: 437-453, 2005

9. Bedoya F, Rubio JC, Morales-Gutierrez C, et al: Single nucleotide change in the cannabinoid receptor-1 (CNR1) gene in colorectal cancer outcome. Oncology 76: 435-441, 2009.

10. Chung SC, Hammarsten P, Josefsson A, et al: A high cannabinoid $\mathrm{CB}(1)$ receptor immunoreactivity is associated with disease severity and outcome in prostate cancer. Eur J Cancer 45: 174-182, 2009. 
11. Michalski CW, Oti FE, Erkan M, et al: Cannabinoids in pancreatic cancer: correlation with survival and pain. Int J Cancer 122: 742-750, 2008.

12. Xu X, Liu Y, Huang S, et al: Overexpression of cannabinoid receptors $\mathrm{CB} 1$ and $\mathrm{CB} 2$ correlates with improved prognosis of patients with hepatocellular carcinoma. Cancer Genet Cytogenet 171: 31-38, 2006.

13. Gustafsson SB, Palmqvist R, Henriksson ML, et al: High tumour cannabinoid $\mathrm{CB} 1$ receptor immunoreactivity negatively impacts disease-specific survival in stage II microsatellite stable colorectal cancer. PLoS One 6: e23003, 2011.

14. Edge S, Byrd D, Compton C, Fritz A, Greene F and Trotti A: AJCC Cancer Staging Manual. Springer, New York, 2010.

15. Jass JR and Sobin LH (eds): Histological Typing of Intestinal Tumours. WHO International Histological Classification of Tumours. 2nd edition. Springer-Verlag, Berlin-New York, 1989.

16. Cianchi F, Papucci L, Schiavone N, et al: Cannabinoid receptor activation induces apoptosis through tumor necrosis factor alphamediated ceramide de novo synthesis in colon cancer cells. Clin Cancer Res 14: 7691-7700, 2008.

17. Jones PA and Baylin SB: The fundamental role of epigenetic events in cancer. Nat Rev Genet 3: 415-428, 2002.
18. Galon J, Costes A, Sanchez-Cabo F, et al: Type, density, and location of immune cells within human colorectal tumors predict clinical outcome. Science 313: 1960-1964, 2006.

19. Naito Y, Saito K, Shiiba K, et al: $\mathrm{CD}^{+} \mathrm{T}$ cells infiltrated within cancer cell nests as a prognostic factor in human colorectal cancer. Cancer Res 58: 3491-3494, 1998.

20. Ropponen KM, Eskelinen MJ, Lipponen PK, Alhava E and Kosma VM: Prognostic value of tumour-infiltrating lymphocytes (TILs) in colorectal cancer. J Pathol 182: 318-324, 1997.

21. Massa F, Marsicano G, Hermann H, et al: The endogenous cannabinoid system protects against colonic inflammation. J Clin Invest 113: 1202-1209, 2004.

22. Shaul YD and Seger R: The MEK/ERK cascade: from signaling specificity to diverse functions. Biochim Biophys Acta 1773: 1213-1226, 2007.

23. Cudaback E, Marrs W, Moeller T and Stella N: The expression level of $\mathrm{CB} 1$ and $\mathrm{CB} 2$ receptors determines their efficacy at inducing apoptosis in astrocytomas. PLoS One 5: e8702, 2010.

24. Malfitano AM, Laezza C, Galgani M, et al: The CB1 receptor antagonist rimonabant controls cell viability and ascitic tumour growth in mice. Pharmacol Res 65: 365-371, 2012. 